\title{
Move Your Wheelchair with Your Eyes
}

\author{
Gökçen ÇETİNEL*1, Sevda GÜL ${ }^{2}$, Zafer TİRYAKi் ${ }^{3}$, Enes KUZU ${ }^{4}$, Meltem MÍLLİGÜNEY ${ }^{5}$
}

Accepted : 12/05/2017 Published: 21/08/2017

DOI: 10.18100/ijamec.2017Special Issue30462

\begin{abstract}
In the proposed study, our goal is to move paralyzed people with their eyes. Otherwise, use this document as an instruction set. Paper titles should be written in uppercase and lowercase letters, not all uppercase. For this purpose, we use their Electrooculogram (EOG) signals obtained from EOG goggles completely designed by the authors. Through designed EOG goggles, vertical-horizontal eye movements and voluntary blink detection are verified by using $5 \mathrm{Ag}-\mathrm{AgCl}$ electrodes located around the eyes. EOG signals utilized to control wheelchair motion by applying signal processing techniques. The main steps of signal processing phase are pre-processing, maximum-minimum value detection and classification, respectively. At first, pre-processing step is used to amplify and smooth EOG signals. In maximum-minimum value detection we obtain maximum and minimum voltage levels of the eye movements. Furthermore, we determine the peak time of blink to distinguish voluntary blinks from involuntary blinks. Finally, at classification step k-Nearest Neighbouring (k-NN) technique is applied to separate eye movement signals from each other. Several computer simulations are performed to show the effectiveness of the proposed EOG based wheelchair control system. According to the results, proposed system can communicate paralyzed people with their wheelchair and by this way they will be able to move by their selves.
\end{abstract}

Keywords: Electrooculogram (EOG), k-Nearest Neighboring (k-NN), wheelchair control.

\section{Introduction}

Electrooculography is a technique for measuring the cornea retinal standing potential that exists between the front and the back of the human eye. Electrooculogram (EOG) signals sourced from the eye movements are used for designing Human Machine Interfaces (HMI), in recent years. Paralyzed people and Amyotrophic Lateral Sclerosis (ALS) patients at later stage lose their moving skills. Moving eye is the last skills of these people. So, HMIs controlled by EOG signals come into prominence day by day.

There are several studies based on EOG and HMI in the literature. In [1], the authors make to control a keyboard with eye movements possible. In this study, EOG signals are measured and then classified by applying k-NN technique. Another study aims to control a mouse with eye movements, again. Signals received offline with wearable eye glasses are used for controlling the curser. Accuracy of this study for blinks, horizontal and vertical saccades is $100 \%, 97 \%$ and $93 \%$, respectively [2]. In [3], an electronic card design that makes EOG signals detectable and useable is explained. The EOG data obtained by this design is displayed and recorded to the computer environment. In on other study, signal processing module that uses a machine learning algorithm and Wavelet Transform to classify the way of eye movements, is demonstrated [4]. In [5], a hand-controllable HCI using eye signals is designed. This application is controlled with accuracy of \%96. In [6], an EOG-based low-cost real-time wheelchair navigation system in which bio-amplifiers and a

\footnotetext{
${ }^{1-5}$ Electrical and Electronics Engineering, Sakarya University, Sakarya54167, TURKEY

* Corresponding Author: Email: gcetinel@sakarya.edu.tr Note: This paper has been presented at the 5th International Conference on Advanced Technology \& Sciences (ICAT'17) held in Istanbul (Turkey), May 09-12, 2017.
}

microcontroller driven servomotor are used, is proposed by the authors. The servomotor is synchronized with the computed eye direction resulted from processing the horizontal EOG signal. In another study, authors try to drive a prototype wheelchair model. The acquired EOG signal is processed to generate control signal depending on the amplitude and duration of signals. In this system, control signals are transmitted in wireless medium [8].

In the proposed paper, EOG signals are obtained by EOG goggles designed by the authors and then processed to control a wheelchair system. EOG goggles measure vertical-horizontal eye movements from two channel and voluntary blink by using five $\mathrm{Ag}-\mathrm{AgCl}$ electrodes. Next, signal processing techniques are performed until the control signal that can move the wheelchair is provided.

The study is organized as follows: In Section II, all stages of the designed circuit used to obtain EOG signals is explained. In Section III, the processing of EOG signals is detailed. In this section, the main features that characterize eye signals are derived and these features are applied to the classifier. Simulation results that show the performance of the proposed system is given in Section III. Finally, Section IV concludes the paper.

\section{Circuit Design}

In our project, we designed an electronic circuit to receive and process real-time EOG signals. The system consists of two channels to detect horizontal and vertical eye movements. The designed circuit consists of five electrodes connected to the amplifier, band pass filter, microcontroller and power amplification as shown in Fig. 1.

The circuit requires symmetrical supply of $\pm 5 \mathrm{~V}$ DC. For this, 7805 and 7905 integrated components used with CA3140 as shown in Fig. 2. In the proposed system this voltage is obtained by a special battery of $18 \mathrm{~V}$. 


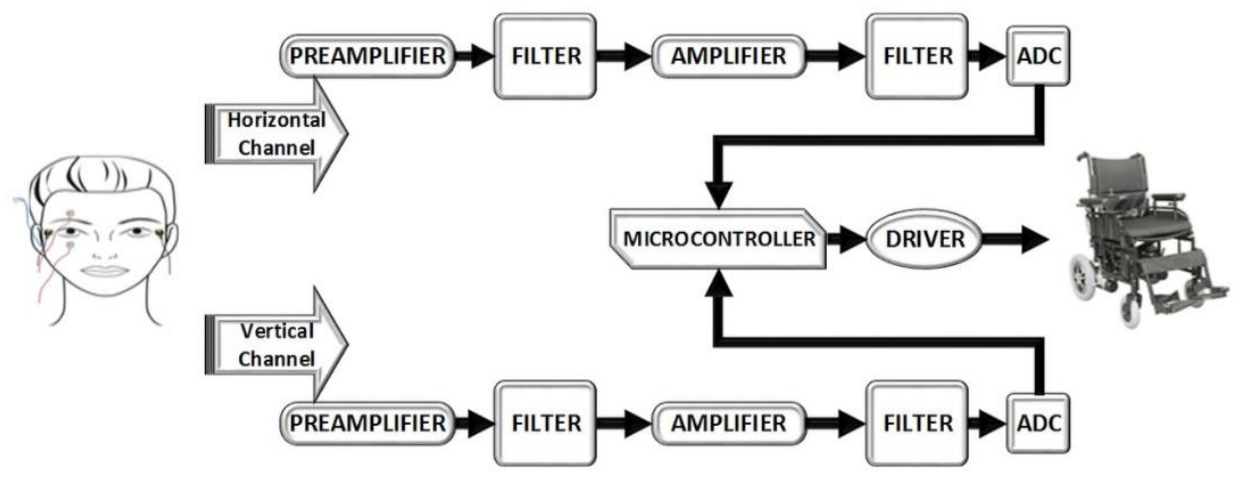

Fig 1. Block Diagram of Proposed System

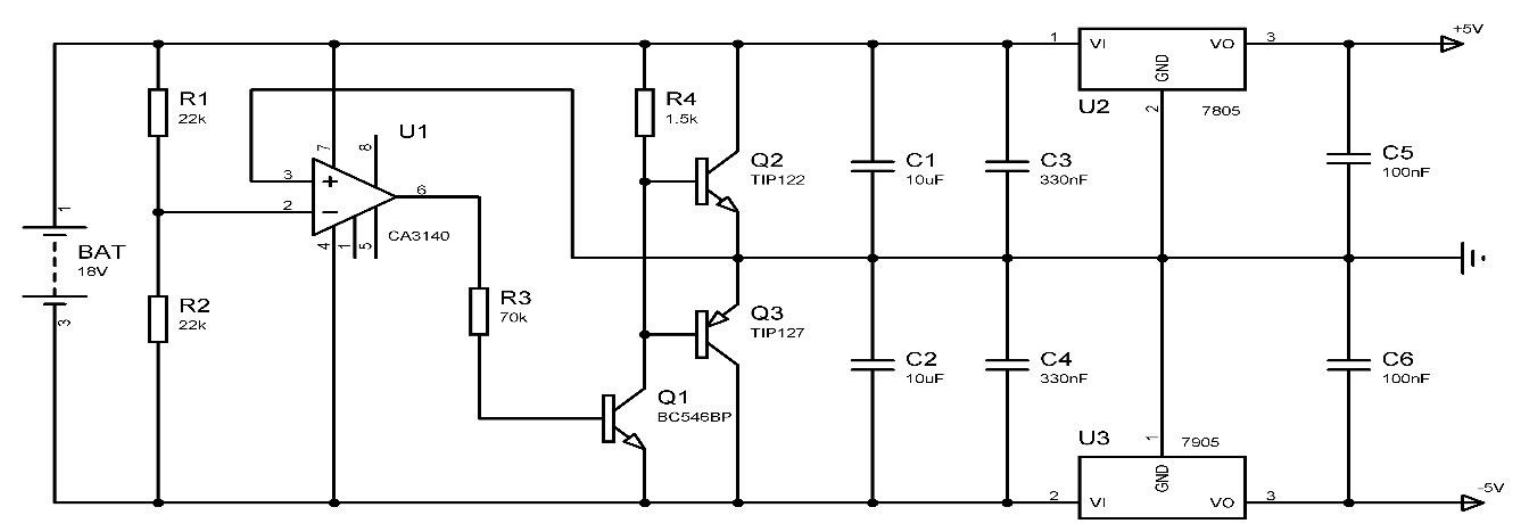

Fig 2. Block Diagram of Proposed System

The main stages or the circuit is given below:

\subsection{Electrode Placement}

Firstly, five $\mathrm{Ag}-\mathrm{AgCl}$ electrodes are located to the patient's face as illustrated in Fig. 3 to measure the EOG signals. In our design we aim to obtain EOG signals in an ergonomic and portable way without disturbing the patient. For this purpose, a portable goggle is designed by the authors. The following factors are taken into account during measuring the eye movement signals.

- $\quad$ Patient / Disabled safety must be provided.

- Electronic noise, especially power line noises, should be suppressed.

- The originality of the biological signal must be preserved.

- Electronic noise and electromagnetic interference should be considered.

\subsection{Amplifying Stage}

The amplitude values of the measured EOG signals are at microvolt levels [8]. Therefore, measured signals need to be subjected to an amplification process in order to process in an easy way. It is seen that this amplification process is difficult to achieve with conventional operational amplifiers when the literature is examined. So, an instrumentation amplifier is utilized for amplification process. In amplification part an AD620 with a high Common Mode Rejection Ratio (CMRR) is used. Gain equation of AD620 is given below [9]:

$$
G=\frac{49.4 k \Omega}{R_{G}}+1 \quad R_{G}=\frac{49.4 k \Omega}{G-1}
$$

\subsection{Filtering Stage}

Other undesired bio-signals detected by the electrodes and the voltage induced to the electrode connection cables are the noise components which disturb the EOG signals in the designed setup. On the other hand, improper placement of the electrodes can also cause noise for EOG signals. To eliminate the effect of noise, filtering process is applied to the measured signals.

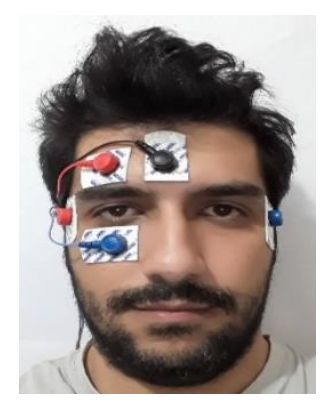

Fig 3. Electrode Placement

The most common used filters for this purpose are high pass, low pass and band pass filters. We used in our project, low-pass filter of which cut-off frequency $0.1 \mathrm{~Hz}$ and high-pass filter with cut-off frequency $3.12 \mathrm{~Hz}$. The portable EOG measurement setup is illustrated in Fig. 4.

\subsection{Microcontroller}

The signals detected from the human body are analog signals. It is clear that analog signals must be converted to digital signals for processing in the computer environment. Analog signals are converted to digital signals by analog digital converters (ADC). In this study, ATmega328P based Arduino UNO card with analog 
inputs and UART module is used. Arduino UNO has 6 ADC inputs with 10-bit resolution. These inputs operate at positive voltages. Therefore, the level shift layer shown in Fig. 5 is added for both channels of the unit.

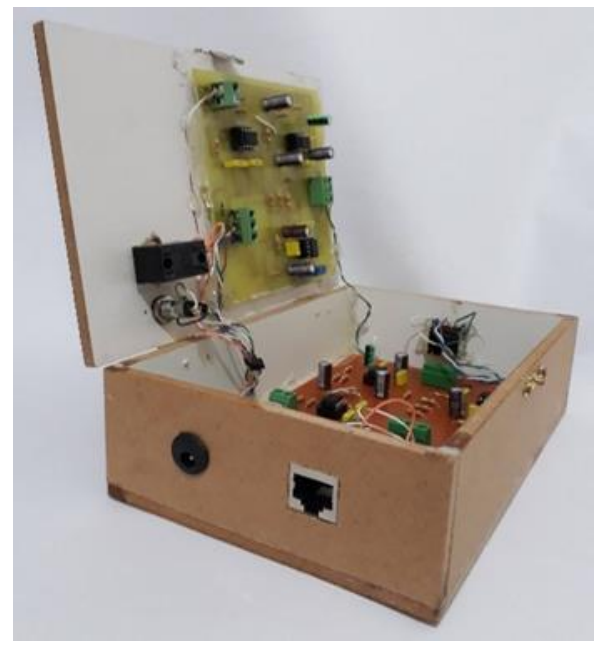

Fig 4. Overview of Portable Circuit

Signals from the horizontal and vertical channels of the circuit are applied to the input of the microcontroller. These channels are represented with $\mathrm{A} 0$ and $\mathrm{A} 1$, respectively. The EOG signals are sampled at $20 \mathrm{~Hz}$ at a sensitivity of $4.88 \mathrm{mV}$. The sampled signals are transferred to the computer via the USB port $[10,11]$.

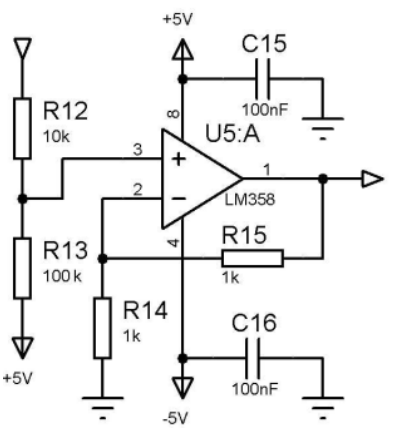

Fig 5. Level Shift Layer [12].

\section{Processing of EOG Signals}

\subsection{Database}

The real-time EOG signals are obtained by the designed circuit and transferred to the computer environment by means of EOG goggles shown in Fig. 6. These specially designed goggles have an adjustable structure to locate the electrodes on proper positions around the eyes for each person. 25 voluntary participants (7 females and 18 males) are included to the study to generate the database. EOG signals are recorded during 125 seconds for each person. Five different signals measured as: left, right, down, up and blink.

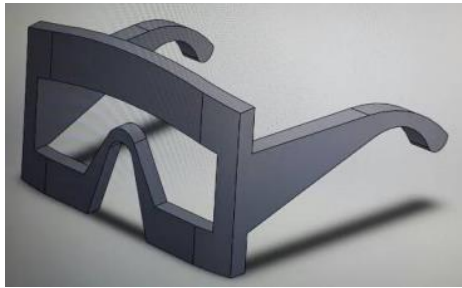

Fig 6. Designed Prototype Goggles

\subsection{Classification and Feature Extraction}

As mentioned above, the database consists of right looking, left looking, up looking, down looking and voluntary blinking movements. Signals corresponding to these eye movements which are received from an individual are shown in Fig 7. Now, we need to determine distinguishing parameters of eye movements to control the wheelchair accurately. This process is referred as feature extraction in signal processing applications. It is known that the stability of the feature extraction phase directly effects the efficiency of the classification phase results.

In the proposed system, five features are derived on the generated database. These features are summarized in Table I. When the eye is moved to right-left or up-down, it forms a signal waveform as illustrated in Fig. 7. As can be seen from the figure these signals have maximum and minimum peaks in different orders. The amplitude values of this peaks and order of the occurrence of maximum and minimum peaks are crucial in determining the characteristic of eye movements.

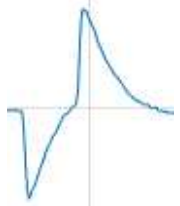

(a)

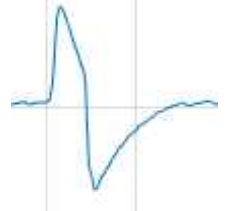

(b)

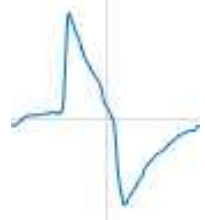

(c)

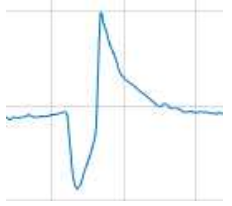

(d)

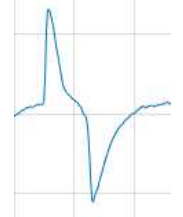

(e)
Fig 7. EOG signals at case of looking four directions and blinking. (a) Right, (b) Left, (c) Up, (d) Down, (e) Blink.

For example, in the first and second rows of the table, minimum and maximum amplitude values of the signal measured from the first channel is given. According to these values we can say that the maximum and minimum amplitude values of the right and left signal are close. So, we need to add another parameter to separate these signals from each other. For this purpose, $\Delta$ parameter is offered. This parameter signs the occurrence order of minimum peak and maximum peak. If we first measured a minimum peak $\Delta$ is +1 . But if we first measured a maximum peak $\Delta$ is -1 . The same explanation can be given for up and down signals which are measured from channel 2. Other parameter given in Table I is $t \Delta$. This parameter is used for distinguishing voluntary blink from involuntary blink. If $t \Delta$ is higher this corresponds to voluntary blink activity.

After deriving true features classification process can be performed. In this study we preferred k-NN technique which is a simple and fast method among the classification methods. To evaluate the performance of the proposed system we use MATLAB program. According to the simulation results, accuracy for right, left, up, down and blink movement is \%100, \%94.4, $\% 93.75, \% 100$ and $\% 98.7$, respectively. 


\section{Conclusions}

In this study, our aim is to design a system in which disabled people can move by their selves. At first phase of the system, the circuit is designed and implemented. Later, portable goggles are designed to be able to receive signals without disturbing the disabled people. The database is generated by measuring EOG signals of 25 voluntary participants. The optimum feature vector is extracted that distinguish five eye movements given before. Finally, by applying k-NN technique, \%98.7 accuracy is achieved for whole system. This results show that the proposed wheelchair control system can be very helpful for disabled people.

Table 1. Feature Extraction for a Signal

\begin{tabular}{|c|c|c|c|c|c|c|}
\hline & Min & $\operatorname{Max}$ & $\Delta$ & $t \Delta$ & Channel & Label \\
\hline Right & 0.9473 & 2.8223 & +1 & 17 & 1 & 2 \\
\hline Left & 0.8350 & 3.3154 & -1 & 12 & 1 & 3 \\
\hline Up & 1.6112 & 2.8516 & -1 & 10 & 2 & 4 \\
\hline Down & 1.0010 & 2.6660 & +1 & 14 & 2 & 5 \\
\hline Blink & 0.7861 & 3.0864 & -1 & 52 & 2 & 1 \\
\hline Min & \multicolumn{6}{|c|}{ : Minimum amplitude value } \\
\hline Max & \multicolumn{6}{|c|}{ : Maximu } \\
\hline$\Delta$ & \multicolumn{6}{|c|}{$\begin{array}{l}\text { : Sign of time difference between max and } \min \\
\text { value. }\end{array}$} \\
\hline $\begin{array}{l}\mathrm{t} \Delta \\
\text { value. }\end{array}$ & \multicolumn{6}{|c|}{ : Absolute value of time difference between max and min } \\
\hline Channel & \multirow{2}{*}{\multicolumn{6}{|c|}{ : Horizontal or vertical data path. }} \\
\hline Label & & & & & \multicolumn{2}{|c|}{ : Classifier label for k-NN. } \\
\hline
\end{tabular}

\section{References}

[1] A. B. Usakli and S. Gurkan, "Design of a Novel Efficient Human; Computer Interface: An Electrooculagram Based Virtual Keyboard," Instrum. Meas. IEEE Trans., vol. 59, no. 8, pp. 2099-2108, 2010.

[2] M. S. Hossain, K. Huda, and M. Ahmad, "Command the computer with your eye - An electrooculography based approach," Ski. 2014 8th Int. Conf. Software, Knowledge, Inf. Manag. Appl., 2014.

[3] M. Y. Esas and F. Latifo, "Bilgisayar Tabanlı İki Kanallı Elektrookülografi Cihazı Tasarımı ve Göz Hareketlerinin Takibi Computer-Based Design with Dual Channel Device Electrooculography and Eye Movement Tracking.", 2015.

[4] A. López, F. J. Ferrero, M. Valledor, and O. Postolache, "EOG Signal Processing Module for Medical Assistive Systems," 2016.

[5] A. Ubeda, E. Ianez, and J. M. Azorín, "An integrated electrooculography and desktop input bimodal interface to support robotic arm control," IEEE Trans. Human-Machine Syst., vol. 43, no. 3, pp. 338-342, 2013.

[6] A. Marjaninejad, Sabalan Daneshvar, "A low-cost Real-time Wheelchair Navigation System Using Electrooculography," no. Icee, pp. 1961-1965, 2014.

[7] B. Champaty, J. Jose, K. Pal, and A. Thirugnanam, "Development of EOG Based Human Machine Interface control System for Motorized Wheelchair," 2014.

[8] R. Barea, L. Boquete, M. Mazo, and E. Lopez, "Wheelchair guidance 'strategies using EOG,” J. Intell. Robot. Syst., vol. 34, no. 3, pp. 279 299, 200.

[9] Analog Device- Low Cost Low Power Instrumentation Amplifier, AD620 Data Sheet.Analog Device Inc. AD620 Datasheet [Online]. Available:http://www.analog.com/media/en/technicaldocumentation/ data-sheets/AD620.pdf

[10] S. Yathunanthan, L. U. R. Chandrasena, A. Umakanthan, V. Vasuki, and S. R. Munasinghe, "Controlling a wheelchair by use of EOG signal,” Proc. 2008 4th Int. Conf. Inf. Autom. Sustain. ICIAFS 2008, no. January 2009, pp. 283-288, 2008.

[11]A. Úbeda, E. Iáñez, and J. M. Azorín, "Wireless and portable EOGbased interface for assisting disabled people," IEEE/ASME Trans. Mechatronics, vol. 16, no. 5, pp. 870-873, 2011.

[12]A. Ayvaz, G. Ertaş, "Design of an EOG Based Electronic Circuit to Control Actuators”, Tiptekno ‘14, pp. 25-28, Kapadokya, 2014. 